\title{
Research on the Non - intelligence Factors of Football Training in Colleges and universities
}

\author{
${ }^{1}$ Shanguo Liu, ${ }^{2}$ Yue Wu \\ ${ }^{1}$ Department of sports rehabilitation and physical education, Changsha \\ Medical University, Changsha, 410219, China \\ ${ }^{2}$ The First Affiliated Hospital of Changsha Medical University, Changsha, \\ 410219, China
}

\begin{abstract}
Football has been widely loved in today's college students group and comment on football is also the main topic of today's college students after school. In today's college sports culture, football itself and the resulting many economic, social and cultural activities are also an important component to have a significant impact on today's people. In addition, the current number of advanced education and teaching theory have agreed that students can achieve the success of learning must be a combination of intellectual and non-intellectual factors. Therefore, the non-intellectual factors of the students are also very important in the study, at the same time, in the current college football teaching, it is further proved that the non-intellectual factors are not only conducive to improving the quality of football teaching itself, more importantly, it effectively help students' healthy growth.

Keywords: non - intelligence factors, football training in colleges and universities
\end{abstract}

\section{Introduction}

With the continuous progress of society, the state of the increasingly demanding of college students, not only requires them to have strong theoretical knowledge, but also requires them to have a strong physique, because good physical quality is to ensure the efficiency of the necessary prerequisite. We need not only limited to 
the strong physique "to enhance physical" surface meaning, and really want to solve the relationship between body and mind. The psychological characteristics of college students are mainly lively, active, strong desire for knowledge, broad interest, but lack of self-control and self-management ability, mental endurance is poor. At present, college students have transition from adolescence to maturity. The development of bodily functions tends to be mature and perfect, and it is the best golden period in the body. In fact, the physical and psychological level are not synchronous, this or that problem, physical development is not only a necessary condition, but also intellectual and moral education an important condition for development. The development of sports interests, to a certain extent, help college students to successfully complete the psychological over time, to protect them more healthy and robust growth.

\section{The non-intellectual factors}

From the source of non-intellectual factors, it first appeared in 1935, the American psychologist Alexander in his paper "specific intelligence and abstract intelligence" for the first time the official "non-intellectual factors" concept put out. By 1950, Alexandria students Wechsler in its "knowledge of innate and nonintellectual wisdom," a paper on the concept of non-intellectual factors were specifically elaborated. Wechsler that the non-intellectual factors, although not replace the basic functions of intellectual factors, but it can largely restrain the intellectual factors.

In the concept, "Ci Hai" on the interpretation of non-intellectual factors are: non-intellectual factors outside the intellectual factors of all psychological factors in general, belong to the human non-cognitive psychological system. Compared with the intellectual factors, non-intellectual factors are broad and narrow sense and generalized non-intellectual factors are mental factors other than intelligence, environmental factors, physiological factors and other psychological factors in general. The narrow sense of non-intellectual factors refers to the performance of people in the behaviour of the more stable personality characteristics, including motivation, needs, interests, emotions, will, personality, temperament and so on.

\section{The football training in colleges and universities}

Football is known as the world's largest sport and it swept the world by the enthusiastic fans around the world sought after, and of course become the first choice for college students to participate in physical exercise. This sport not only drives people's motor nerves, but also promotes economic, social and other aspects of development, which are for people's daily life has played a very important role, but also brought a wide range of impact. These effects also contribute to the development and progress of football. It is precisely because the world football culture is so popular, it makes the college football teaching has 
become an integral part of the campus sports culture. Since college students interested in the sport of football so strong, physical education teachers should pay attention to the rationality of teaching methods, students should pay attention to all aspects of training. In these aspects of education and training, the general to grasp the two factors, namely, intellectual factors and non-intellectual factors, which students can achieve good academic performance of the key two points. In the university physical education teaching, especially the soccer teaching process, the non-intellectual factor cultivation is very important. Football education experience shows that the cultivation of non-intellectual factors not only conducive to the improvement of the quality of teaching football, but also conducive to the lifelong development of students.

\section{The importance of cultivating college students' non- intelligence factors}

It is found that non-intelligence factors can not only influence and promote each other, but also play a unique role in cultivating the quality of college students.

\subsection{Make college students establish firm motives and beliefs}

With the development of the market system, the value orientation and motivation of the college students are becoming more and more diversified. Many college students in the early and high school students into the key high school, college, wait until after entering the university, due to the significant reduction in the pressure of college students, college students there will be significant success motivation uncertainties. In the face of fierce social competition, the lack of social experience of college students do not know how to develop, which led to many college students there is a quick individualistic tendencies. Cultivating the non-intellectual factors of college students can make college students establish a firm motive of success, which is also the basis for college students to enter the society in the future to obtain good results of personal activities.

\subsection{Focus on the cultivation of college students' interest}

Focus on the cultivation of college students' interest is an important measure of non-intellectual factors. The great scientist Albert Einstein once said: "Interest is the best teacher of mankind", that is, only one person interested in a thing, so he will take the initiative to knowledge, exploration and practice, and knowledge, exploration and Practice of the process, resulting in a sense of pleasure, for its further study of the things laid the foundation. Because of this, many educators at home and abroad have attached great importance to the development of student interest. Take a student's interest in a course as an example. If a student is interested in this course, he or she will be motivated and willing to spend time and energy in the course. Not only is it easier to learn, But also relatively easy to achieve good results. 


\section{3 Enable students to maintain positive emotions and emotions}

Emotions and emotions are an obvious external indicator and it is the psychological activities of people often play a role in promoting or repressing. Optimistic, cheerful, excited people thinking agile, memory enhancement, full of confidence; depression, pessimism, dull lead to thinking inhibition, memory difficulties and pessimistic disappointment. In the mood for love is the college students, their emotional experience is very rich, emotional ups and downs are also large, which is one of the outstanding characteristics of young people. Cultivating college students' non - intelligence factors can keep college students' good emotional state, so that they can withstand setbacks and avoid complacency, which will make college students' emotion develop healthily. College students have this healthy emotional state, will be able to abundant energy, keen observation, creative learning, it will make their own mental health development.

\subsection{It can cultivate university students' perseverance}

Among all the winners, there is no perseverance, firmness is not strong, have played a decisive role. Su Shi, the ancient Chinese writer once said: "The ancient legislature event, not only super-talented, there must be perseverance," shows that the cultivation of perseverance is an important guarantee for college students. Cultivating college students 'non - intelligence factors can make college students' perseverance develop effectively. Modern people generally bear more pressure than the past, college graduates into society, will face all kinds of difficulties, if the willpower is weak, it is likely to be overwhelmed by these difficulties, on the contrary, if you have a strong will Force, will be able to face up to difficulties, perseverance in the pursuit of their own goals, which is an important foundation for college students.

\section{The training strategy of non-intellectual factors in college football training}

Human non-intellectual factors can be acquired through the day to get the appropriate upgrade. In college football teaching, the following ways and strategies can be used to train students of non-intellectual factors.

\subsection{Take encouragement mechanism}

We often say that interest is the best teacher. In college football teaching, student interest is a prerequisite for successful teaching. To stimulate students to actively participate in the enthusiasm of football, so that they develop good exercise habits, which are the college football teachers should do one aspect. Some students because of football's basic technology is not solid, poor physical condition, poor health, and therefore the enthusiasm for football is not high, and even negative and contradictory emotions. These are the students of non- 
intellectual factors immature performance. Once the students have such problems, teachers should be appropriate to change their teaching methods. Students can use different teaching methods, such as divided into sports players, team members, referees and so on, so that students in the football field can play their own advantages, in the whole groups do not ignore every student, so as to fully mobilize the enthusiasm of students, but also for them to establish their confidence, play to their strengths, to achieve the purpose of common improvement. This non-intellectual factors for students to develop very effective.

\subsection{Establish students self-confidence}

Football in the technical and tactical is very complex, and football requires a strong self-confidence to complete a series of tactical moves. In the field when the game, the two teams' battle situation is changing rapidly and some time Emmanuel Zhaxian, perhaps the best chance. This opportunity to grasp the fleeting, if there is no strong self-confidence, a lot of passing action, shooting action, defensive action will appear not determined, not decisive, it wasted attack the best time. This is not worth the athletes. Therefore, in order to win the game victory, the athlete's self-confidence is necessary to train. In the process of college football teaching is the same.

\subsection{Cultivate a sense of competition.}

Football itself is a very confrontational campaign, in this highly competitive movement, the students' sense of competition must be improved. This intense competition requires students to maintain a high degree of vigilance during the time, in order to ultimately win the game. Therefore, the college football teachers should cultivate the students' sense of competition into the agenda, according to the characteristics of their own teaching students in the class to create a competitive atmosphere, so that students can truly understand the dynamics of football this alienation, on the other hand In the sports skill movement exercises require a higher project practice, due to physical condition and many other limitations, resulting in unsatisfactory results. Although these students are very fond of physical education, but participate in sports activities, self-confidence, it alienated sports activities. Then how to make these students like other students can be active and active into the physical exercise and fun, is the responsibility of each of our physical education teachers and the pursuit of goals. Through teaching practice, I realized that as long as the teachers to care for different types of students, the use of more advanced teaching evaluation method for students to establish self-confidence in physical education, have their own income, can well promote the students participate in sports, So that students with life and health and health in parallel. 


\section{Conclusion}

In college soccer teaching, some soccer games are arranged according to the characteristics of college students. In the college physical education teaching, there is a kind of intense atmosphere in which the students can devote themselves to football and give full play to their potential. In the teaching process, we should pay attention to cultivating students to comply with the rules of equal quality, we should pay attention to the cultivation of competitive awareness of students at the same time, so that students can learn how to keep peaceful coexistence.

\section{References}

[1] Non - intelligence factors and innovation of physical education teaching, 55(12), pp.68-70, 2011

[2] Yan Hua, Dong Xinwei, Liu Xiaodong, Analysis on the Function and Cultivation of Non-intelligence Factors in Football Teaching. Acta Metallurgica Sinica, 8 (5), pp.87- 91, 2013

[3] Xie Liming. Research on Physical Education of College Students. Journal of Nanjing Institute of Physical Education, 12(10), pp. 18- 20, 2012

[4] Shen Deli. Non-intellectual factors and personnel training. Beijing: Education Science Press, pp.58- 61, 1992

[5] Wang Xiaohua, Zeng ZhaoHao. Discussion on the Importance of College Students' Non-intelligence Factors Journal of Liaoning Administration College 8(4), pp.85-87, 2014 